\title{
Fruit and vegetables consumption patterns and risk of chronic disease of lifestyle among university students in Kenya
}

\author{
A.M. Nyanchoka ${ }^{1}$, M.E. Van Stuijvenberg ${ }^{2}$, A.B. Tambe ${ }^{1}$ and X.G. Mbhenyane ${ }^{1}$ \\ ${ }^{1}$ Division of Human Nutrition, Faculty of Medicine and Health Sciences, Stellenbosch University, Cape Town, \\ South Africa and \\ ${ }^{2}$ Non Communicable Diseases Research Unit, South African Medical Research Council, Cape Town, South Africa
}

The nutrition transition in developing countries has contributed to the significant early onset of Chronic Diseases of Lifestyle (CDLs) in the adult population at a younger age ${ }^{(1)}$. CDLs including type 2 diabetes, accounts for $27 \%$ of deaths suffered by Kenyans, and the likelihood of dying too young from a CDL in Kenya is $18 \%{ }^{(2,3)}$. The aim of this study was to assess the fruit and vegetable consumption patterns and risk of CDLs among university students aged 19-30 years.

A cross-sectional analytical design was used to randomly recruit 423 self-catering subjects (168 males; 255 females) aged 19-30 years attending Kenyatta University in Kenya. The sample was representative of all faculties in the University, and all regions in Kenya. Fruit and vegetable consumption patterns were measured using a fruit and vegetable intake questionnaire and a food frequency questionnaire. Physical and biochemical measures were used to assess the risk factors of chronic diseases of lifestyle among the participants. Data were analysed by IBM SPSS Version 26.0.

The proportion $(95 \% \mathrm{CI})$ of respondents who met the WHO recommendation of 5 or more servings of fruits and/or vegetables per day in a typical week was $21.5 \%$. The mean intake of fruits was 1.7 servings per day and for vegetables, 1.9 servings per day. The prevalence of risk factors of CDLs: $28.1 \%$ of the respondents are current consumers of alcohol; $4.0 \%$ current tobacco users; $18.2 \%$ were overweight (BMI); $16.5 \%$ had abdominal obesity (WC); 9.5\% had high WHR; $8.3 \%$ had raised blood pressure, and $39.5 \%$ had raised cholesterol. A significant association between meeting the WHO recommendation of 5 or more servings of fruits and/or vegetables in a day and combined risk factors of CDLs $(\mathrm{p}=0.0001)$.

Over $78 \%$ of the study population consumed lower amounts of fruits and vegetables than recommended by the WHO. Eighty-five percent had at least one combined risk factors of CDLs. There is a need to develop and strengthen nutrition campaigns aimed at increasing fruit and vegetable intake levels among young adults, and the prevention of chronic diseases of lifestyle.

\section{Acknowledgments}

This research was funded by the National Research Foundation (NRF) - South Africa

\section{References}

1. Vorster HHE, Bourne LT. The Nutrition Transition in Developing Countries. In Community Nutrition for Developing Countries. AU Press, and Unisa Press. p54-62

2. WHO. UN, Kenyan government take broad-based approach to fighting NCDs. Available:http://www.who.int/nmh/events/2014/ kenya-ncd-prevention/en

3. Ministry of Health Kenya. KENYA STEPwise survey for non-communicable disease risk factors 2015 report, Nairobi 